\title{
Muscle development in dendrobranchiate shrimp, with comparison with Artemia
}

\author{
Daniel A. Kiernan and Philip L. Hertzler* \\ Department of Biology, Central Michigan University, Mount Pleasant, MI 48859, USA \\ *Author for correspondence (email: Philip.L.Hertzler@cmich.edu)
}

SUMMARY The muscle pattern of malacostracan and entomostracan crustacean nauplius larvae was compared using fluorescent phallotoxins. In the dendrobranchiate malacostracan Sicyonia ingentis, F-actin staining was first detected in limb setae at $12 \mathrm{~h}$, likely within sensory nerves. Staining of $\mathrm{F}$-actin was detected in the trunk at $15 \mathrm{~h}$ and grew into the naupliar limbs. Sarcomeres were detected at $19 \mathrm{~h}$, identifying the structures as extrinsic limb muscles. The extrinsic limb muscles enlarged but retained their general pattern during the later nauplius stages. Longitudinal trunk muscles and circumferential visceral muscle (VM) developed in the post-naupliar region during nauplius instars 4 and 5 , at the time when the gut also formed. In the anostracan branchiopod Artemia salina, the newly hatched nauplius contained an extensive system of extrinsic and intrinsic limb muscles. The gut was almost complete at hatching, along with its associated circumferential VM. Muscles similar in position and structure could be identified in nauplii from the two taxa, but different anatomical origins of extrinsic muscles were evident. Whether the naupliar limb muscles are homologous in malacostracans and branchiopods remains an open question. The strong musculature of the dendrobranchiate naupliar limbs correlates with the use of all three pairs of limbs for swimming.

\section{INTRODUCTION}

The nauplius has been recognized as a fundamental crustacean larval stage since the demonstration by J. Vaughan Thompson in 1829 that barnacles produced nauplius larvae and were therefore crustaceans (reviewed in Anderson 1994). Later, F. Müller discovered the nauplius larvae in dendrobranchiate shrimp, members of the largest and most diverse crustacean group, the Malacostraca. Müller (1864) used the nauplius larva as the basis of his argument for a recapitulation theory of arthropod origins, before Haeckel (reviewed in Bowler 1994). Although the relationships among the crustacean taxa are still unclear, recent work has clarified the phylogenetic relationships among the Malacostraca (Jarman et al. 2000; Richter and Scholtz 2001). A free-swimming nauplius stage is present in most basal groups of crustaceans, leading some to identify the nauplius as a crustacean phylotypic stage (Williams 1994; Dahms 2000). However, a free nauplius (FN) is rarely found among the Malacostraca, with the Euphausiacea and decapod Dendrobranchiata the only groups where this is so. In most malacostracans, development proceeds through an embryonic "egg-nauplius" stage to hatching and release at a more advanced larval stage with additional segments and limbs (Strathmann and Eernisse 1994; Scholtz 2000). In the most parsimonious interpretation of the available evidence, which we will assume here, the egg nauplius (EN) is likely the ancestral condition for the Malacostraca, with the FN being secondarily derived (Scholtz 2000).

The presence of a FN in the Euphausiacea and Dendrobranchiata presents a puzzle: either the EN evolved multiple times in the Malacostraca from an ancestral FN condition or there was a reversion to a FN in the euphausiacean and dendrobranchiate lines from an ancestral EN condition (Strathmann and Eernisse 1994; Scholtz 2000). In the former hypothesis, the FN is the plesiomorphic condition for the Malacostraca, with multiple apomorphic occurrences of the egg nauplius. In the second hypothesis, the EN is the plesiomorphic condition for the Malacostraca, with secondary reversion to the $\mathrm{FN}$ in euphausiaceans and dendrobranchiates (Scholtz 2000). This interpretation is clearly more parsimonious (Scholtz 2000; Richter and Scholtz 2001) and will be assumed here. Now the question becomes: did the reversion to the FN occur once or twice? The answer to this depends on the phylogenetic position of the Euphausiacea (Alwes and Scholtz 2004). Traditionally, the Euphausiacea have been grouped with the Decapoda to form Eucarida (Fig. 1A), whereas more recent proposals (Jarman et al. 2000; Richter and Scholtz 2001) group the Euphausiacea with the Peracarida (Fig. 1B).

The reversal to the FN likely involved a novel deployment of genes responsible for patterning the nauplius body plan, so 

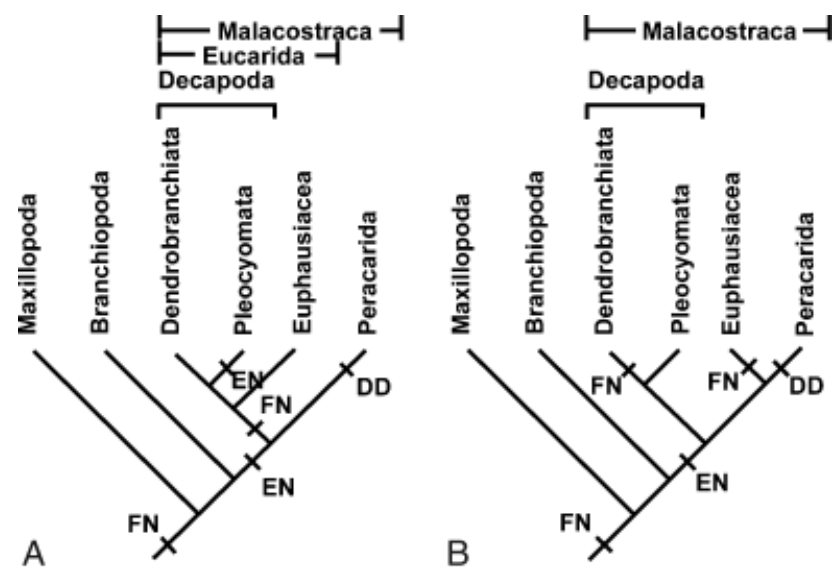

Fig. 1. Larval types mapped onto two phylogenies of the Crustacea. (A) Euphausiacea-Decapoda sister relationship (Eucarida). (B) Euphausiacea-Peracarida sister relationship. The free nauplius (FN) is shown as an autapomorphy of the Eucrustacea, relative to the Arthropods as a whole (Walossek 1999). The appearance of an egg nauplius (EN) in the stem line of the Malacostraca is shown as an apomorphy within the Crustacea, plesiomorphic for the Malacostraca. A reversion to a free nauplius has occurred either once with loss in the Pleocyomata and other malacostracans (A) or twice independently in the Dendrobranchiata and the Euphausiacea (B). Direct development (DD), not excluding passage through an EN, has evolved in the Peracarida (Scholtz 2000).

that the anatomical details might differ from nauplius larvae in basal crustaceans such as branchiopods. To begin to study this question, more data are needed on how free-swimming nauplii develop in the Dendrobranchiata and Euphausiacea as compared with basal crustaceans. We have focused on the skeletomuscular system. The work of Hessler (1964) and Manton $(1964,1977)$ laid the foundation for the analysis of crustacean muscle systems. Manton established the homologies of adult mandible muscles and their modification for diverse modes of action. Crustacean myogenesis has been best described in barnacles (Maxillopoda: Cirrepedia) and brine shrimp (Branchiopoda: Anostraca; reviewed by Anderson 1973). In the cirripede Tetraclita rosea, muscle development begins with the formation of three naupliar somites, solid blocks of mesodermal tissue within the first antennal, second antennal, and mandibular segments (Anderson 1969). The somites give rise to the somatic limb musculature of the nauplius, which propels itself by oar-like swimming movements of the naupliar appendages. The somatic naupliar muscle anatomy is carefully described for the cirripede Balanus balanoides (Walley 1969). Naupliar trunk muscle anatomy has also been described for the anostracan branchiopods Artemia salina (Benesch 1969) and Branchinecta ferox (Fryer 1983). A complex limb musculature underlies the multi-branched limbs of the notostracan branchiopod Triops longicaudatus (Williams and Müller 1996). Staining with fluorescent phallotoxin re- vealed that muscle formation occurs subsequent to the folding and subdivision of the simple limb bud.

Among decapod malacostracan larvae, abdominal muscle development was described in zoea and mysis larvae of the caridean shrimp Crangon vulgaris (Daniel 1930). Numerous studies have examined the larval stages of dendrobranchiate shrimp (see citations in Dall et al. 1990) but few have analyzed how the internal structures arise during development. Mesoderm formation has been described in Penaeus kerathurus (Zilch 1978, 1979) and Sicyonia ingentis (Hertzler and Clark 1992; Hertzler 2002), where naupliar mesoderm arises early in cleavage whereas teloblastic mesoderm forms later from the ventral mesendoblast. The adult muscles of the dendrobranchiate shrimp Penaeus (Litopenaeus) setiferus have been carefully examined (Young 1959) and provide an ultimate endpoint for this study.

The first objective of this study was to trace the appearance of muscle from the embryo through the nauplius stages in the Dendrobranchiata, as part of an effort to describe myogenesis from the egg to the adult in this group. Using fluorescently labeled phallotoxins and confocal microscopy, we examined the development of the naupliar muscle in dendrobranchiate shrimp. The second objective was to compare the naupliar muscles of dendrobranchiates, representing the Malacostraca, with those of Artemia (Branchiopoda: Anostraca), representative of the nonmalacostracan crustaceans.

\section{MATERIALS AND METHODS}

\section{Sicyonia embryos and larvae}

Sicyonia ingentis were collected by otter trawl near San Pedro, CA, and transported to Occidental College during the summer of 2001. Gravid females were maintained in a temperature-controlled 100-1 tank with recirculating seawater and induced to spawn as in Pillai et al. (1988). Animals were spawned into finger bowls containing artificial seawater (ASW, $\mathrm{pH}$ 8.2), prepared according to Cavanaugh (1956) and containing $50 \mu \mathrm{M} 3$-amino-1,2,4,-triazole (ATA) to facilitate the removal of the hatching envelope. Eggs were swirled for $5 \mathrm{~min}$ while the jelly was released, checked for the presence of bound sperm, and cultured at $22^{\circ} \mathrm{C}$. At $20 \mathrm{~h}$ post-spawning, embryos were transferred to ASW in separatory funnels with oxygenation. Tetracycline was added to $0.2 \%$ and ozonation was provided to control bacterial growth. Embryos were collected every hour from 10 to $23 \mathrm{~h}$ post-spawning, and nauplius larval stages were collected over a period of 3 days.

\section{Artemia larvae}

Great Salt Lake origin A. salina cysts were obtained from Brineshrimpdirect.com and oxygenated in ASW for $1 \mathrm{~h}$. Cysts were treated with $100 \%$ bleach for $1 \mathrm{~min}$ to remove envelopes, and then washed with tap water and $1 \%$ sodium thiosulfate to remove residual bleach. Cysts were transferred to ASW and cultured in 1-1 separatory funnels with oxygenation. Larval stages were collected every $12 \mathrm{~h}$ from hatching, beginning at $22 \mathrm{~h}$ following rehydration. 


\section{Fixation and fluorescent-phallotoxin staining}

Sicyonia embryos were passed through $200 \mu \mathrm{m}$ Nitex nylon screening to remove hatching envelopes and fixed in $4 \%$ paraformaldehyde in ASW for $2 \mathrm{~h}$ at $22^{\circ} \mathrm{C}$. Fixed samples were washed and briefly stored in ASW at $4^{\circ} \mathrm{C}$ until staining. Late-stage embryos and nauplii were placed in glass vials containing ASW and sonicated using a Branson 1510 tub sonicator for $3 \mathrm{sec}$ to permeabilize the exoskeleton. Samples were stained with BODIPY FL-conjugated phallacidin (Molecular Probes Inc., Invitrogen, Carlsbad, CA, USA) diluted to $6.6 \mu \mathrm{M}$ in ASW for $1.5 \mathrm{~h}$ with gentle agitation in the dark. Samples were then washed with ASW and passed through a dehydration series to $100 \%$ ethanol. Samples were finally transferred to methyl salicylate for clearing and mounting.

Artemia larvae were fixed in $4 \%$ paraformaldehyde in phosphate-buffered saline (PBS), $\mathrm{pH} 7.0$, for $2 \mathrm{~h}$ at $22^{\circ} \mathrm{C}$. Fixed samples were washed and stored temporarily in PBS. Samples were sonicated and stained with BODIPY FL- conjugated phallacidin as above. After washing with PBS, samples were dehydrated, cleared, and mounted as above. Stained specimens were imaged with the argon laser on the Olympus Fluoview 300 laser scanning confocal microscope (Olympus America Inc., Center Valley, PA, USA) at Central Michigan University. Images were collected using Planapo 20X 0.7 NA, Planfluor 40X 0.75 NA, or Planapo 60X 1.4 NA objectives, and 3-D reconstructions and measurements were performed using the Fluoview software. Plates were prepared using Adobe Photoshop 7.

\section{RESULTS}

\section{Appearance of F-actin in Sicyonia embryos}

Staining of Sicyonia embryos with phallacidin revealed no F-actin staining through $11 \mathrm{~h}$ post spawning (Fig. 2A). Foci of F-actin staining appeared at $12 \mathrm{~h}$ in the pre-antennulary region (PAR), the first antennae (A1), the second antennae (A2), the mandibles (Mn), and the post-naupliar region (PNR; Fig. 2B, arrowheads). The pre-antennulary foci persisted through $15 \mathrm{~h}$ but did not develop further. Two A1 foci were present near the distal tip and one at the posterior margin of the limb bud. Several foci were observed throughout the A2 (Fig. 2B). Four foci were seen in the Mn, with two near the distal tip and two toward the middle of the Mn. Two PNR foci were also present, where the furcal spines eventually develop (Fig. 2B). In the 13-h embryo, staining of F-actin had extended toward the trunk from more densely staining regions distally (Fig. 2C). F-actin staining was also observed in the post-naupliar region of the embryo, on either side of the blastopore (Fig. 2C, BLP). Four foci appeared in a rectangular formation in the dorsal trunk (Fig. 2C, arrowheads). At $14 \mathrm{~h}$ post spawning, F-actin extended further across the limb buds (Fig. 2D). By $15 \mathrm{~h}$, a new pattern of F-actin staining appeared in the A2 trunk region (Fig. 2E). Higher magnification revealed no striations in the F-actin staining either in the limbs or trunk. The origin of the limb F-actin fibers in the ectoderm, their growth toward the trunk, and their elongated nature suggest that they are within sensory nerve cells associated with the setation of the larva.

By $16 \mathrm{~h}$, trunk F-actin had extended inward toward the midline from the bases of the A1 and A2 limbs (Fig. 2F, arrowheads). F-actin continued to extend toward the proximal limb and distally out into the terminal setae (Fig. 2F, TS). At $17 \mathrm{~h}$, three bands of F-actin staining were anchored at the dorsal midline in the A2 segment of the embryo (Fig. $2 \mathrm{G}$, arrowheads). Dorsal Mn segment F-actin ran from the proximal end of the Mn appendage into the Mn appendage (Fig. $2 \mathrm{G}, \mathrm{Mn}$ ). Further extension of trunk and limb F-actin occurred by $18 \mathrm{~h}$ with additional staining associated with the furcal spines of the post-naupliar region (Fig. $2 \mathrm{H}$, arrowhead). By 19 h, F-actin appeared throughout the limbs, and the staining in the trunk extended further distally (Fig. 2I, arrowheads). A higher magnification at $19 \mathrm{~h}$ showed that sarcomeres were now present in the trunk staining (Fig. 2J), but not in the ends of the limbs (not shown). Therefore, the Factin staining in the trunk was extrinsic limb muscle (ELM). At $20 \mathrm{~h}$, ELM extended from the midline of the A2 segment and from the sides of the Mn segment (Fig. 2K). Further proliferation of ELM was seen at $22 \mathrm{~h}$. F-actin staining also extended up to the Mn segment in the post-naupliar region (Fig. 2L, arrowheads).

Dorsal and ventral regions of muscle attachment to the midsection became clearly apparent with the lengthening of the body at $23 \mathrm{~h}$ (Fig. 3A). Dorsal trunk fibers extended into the $\mathrm{A} 1$ and $\mathrm{A} 2$ appendages whereas the $\mathrm{Mn}$ appendages received dorsal trunk fibers near their proximal ends. Ventral ELM extended into all three naupliar appendages (Fig. 3A). Muscle contractions began in the limbs at this time point, before emergence of the nauplius from the hatching envelope.

\section{Muscle development in Sicyonia nauplius I-V}

At $24 \mathrm{~h}$, the fully developed nauplius I larva broke through its encasement, the hatching envelope. Dorsal and ventral muscles were now functional for limb movement and swimming (Fig. 3B). The nauplius II stage was reached by $40 \mathrm{~h}$ (Fig. 3C). The nauplius body had lengthened further and ELM thickened in both the appendages and trunk area. A single optical section revealed the first development of the gut epithelium at this stage (Fig. 4A, arrowheads). At $50 \mathrm{~h}$, the nauplius III had thickened ELM, and F-actin staining in the post-naupliar region and around the stomodeum (Fig. 3D, STM). A dorsal section showed continued development of the digestive system (Fig. 4B, arrowheads). Endodermal yolk cells (EYC) remained inside the gut epithelium at the nauplius II and III stages. The proctodeum (PRM) was seen clearly in a ventral section with foci of F-actin staining to the left and right (Fig. 4C, arrowheads).

Posterior longitudinal muscle (PLM) extended into the maxillary segments of the nauplius IV, around $75 \mathrm{~h}$, and the 

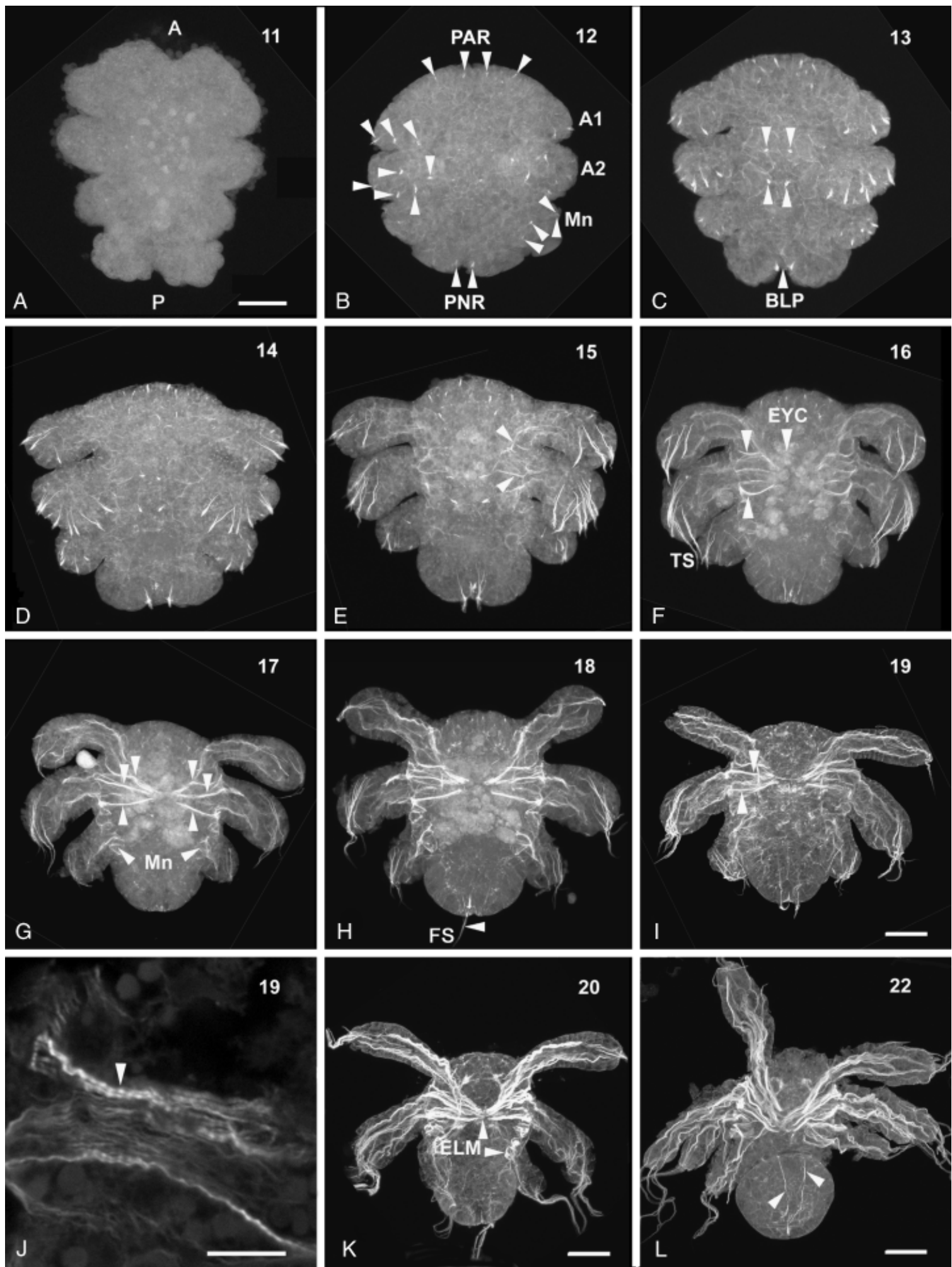

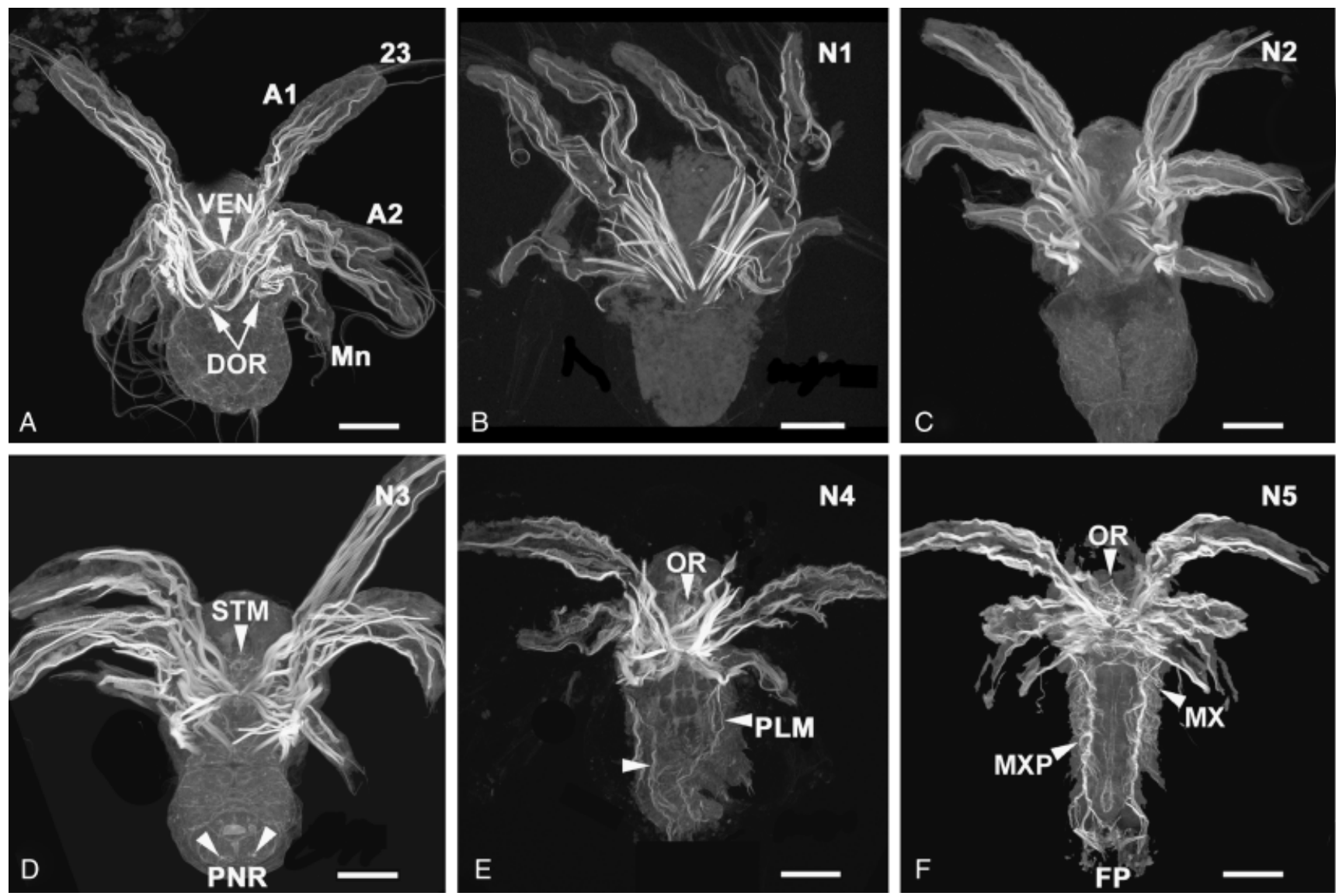

Fig. 3. Overview of muscle development in Sicyonia $23 \mathrm{~h}$ embryo and nauplius larval stages I-V. Each image is a composite of optical sections through an entire embryo, stained with BODIPY FL-phallacidin, oriented with anterior at the top. (A) Prehatch nauplius I at $23 \mathrm{~h}$, when muscle contractions begin, showing dorsal (DOR) and ventral (VEN) muscle origins. (B) Hatched nauplius I at $28 \mathrm{~h}$ showing fully functional limb muscle for swimming. (C) Nauplius II at $40 \mathrm{~h}$ showing extension of longitudinal muscle in both the appendages and trunk area. (D) Nauplius III at $50 \mathrm{~h}$ showing first signs of circumferential muscle appearance around the stomadeal opening (STM) and F-actin staining in the post-naupliar region (PNR). (E) Nauplius IV at $75 \mathrm{~h}$ revealing extension of longitudinal muscle into the posterior region (PLM) and oral region muscle (OR). (F) Nauplius $\mathrm{V}$ at $84 \mathrm{~h}$ showing extensive proliferation of longitudinal muscle in the posterior furcal process $(\mathrm{FP})$ and oral regions $(\mathrm{OR})$ and extension of limb muscle into the maxillae $(\mathrm{MX})$ and maxillipeds $(\mathrm{MXP}) . \mathrm{Scale}$ bar $=50 \mu \mathrm{m}$.

Fig. 2. Overview of muscle development in Sicyonia embryos from 11 to $22 \mathrm{~h}$. Except for panel J, each image is a composite of optical sections through an entire embryo, stained with BODIPY FL-phallacidin, oriented with anterior at the top. Panel J shows a single confocal section at high magnification. Time in hours is noted in the upper right of each panel. (A) Phallacidin staining revealed no F-actin through $11 \mathrm{~h}$ post spawning (A, anterior; $\mathrm{P}$, posterior). (B) F-actin is first detected by $12 \mathrm{~h}$, in several foci (arrowheads) within the pre-antennulary region (PAR), antennules (A1), antennae (A2), mandibles (Mn), and post-naupliar region (PNR). (C) Areas of F-actin staining have increased in length by $13 \mathrm{~h}$, growing from a thickened distal end. Four additional foci appear in the trunk (arrowheads). Two PNR foci are adjacent to the blastopore (BLP). (D) By $14 \mathrm{~h}$, three major areas of F-actin staining are evident in A1, seven within A2, and four within the Mn segment. Growth continues from the posterior foci toward the anterior. (E) By $15 \mathrm{~h}, \mathrm{~F}$-actin has formed within the trunk (arrowheads), whereas growth continues proximally in the limbs as they grow distally. $(\mathrm{F})$ By $16 \mathrm{~h}$, a series of F-actin stained bands are present mediolaterally within A2 trunk (arrowheads). Endodermal yolk cells (EYC) stain above background. (G) By 17 h, three major A2 segment F-actin-stained bands converge on the dorsal midline (arrowheads). Additional F-actin staining is present in the proximal end of the mandible $(\mathrm{Mn}) .(\mathrm{H}) \mathrm{By} 18 \mathrm{~h}$, the $\mathrm{F}$ actin within the trunk extends into the limbs and associates with F-actin in the limbs whereas F-actin in the PNR is associated with the furcal spines (FS). (I) By $19 \mathrm{~h}$, striations corresponding to sarcomeres could be observed in the F-actin filaments within the trunk (arrowheads), but not at the distal ends of the limbs. (J) Higher magnification of trunk F-actin staining, showing first appearance of sarcomeres (arrowhead). (K) By $20 \mathrm{~h}$, the trunk F-actin fibers have extended into the limbs, defining them as extrinsic limb muscles (ELM). (L) At $22 \mathrm{~h}, \mathrm{~F}$-actin staining in the PNR likely is within nerves to the furcal spines (arrowheads). Panel $\mathbf{J}$ scale bar $=10 \mu \mathrm{m}$; all other scale bars $=50 \mu \mathrm{m}$. 

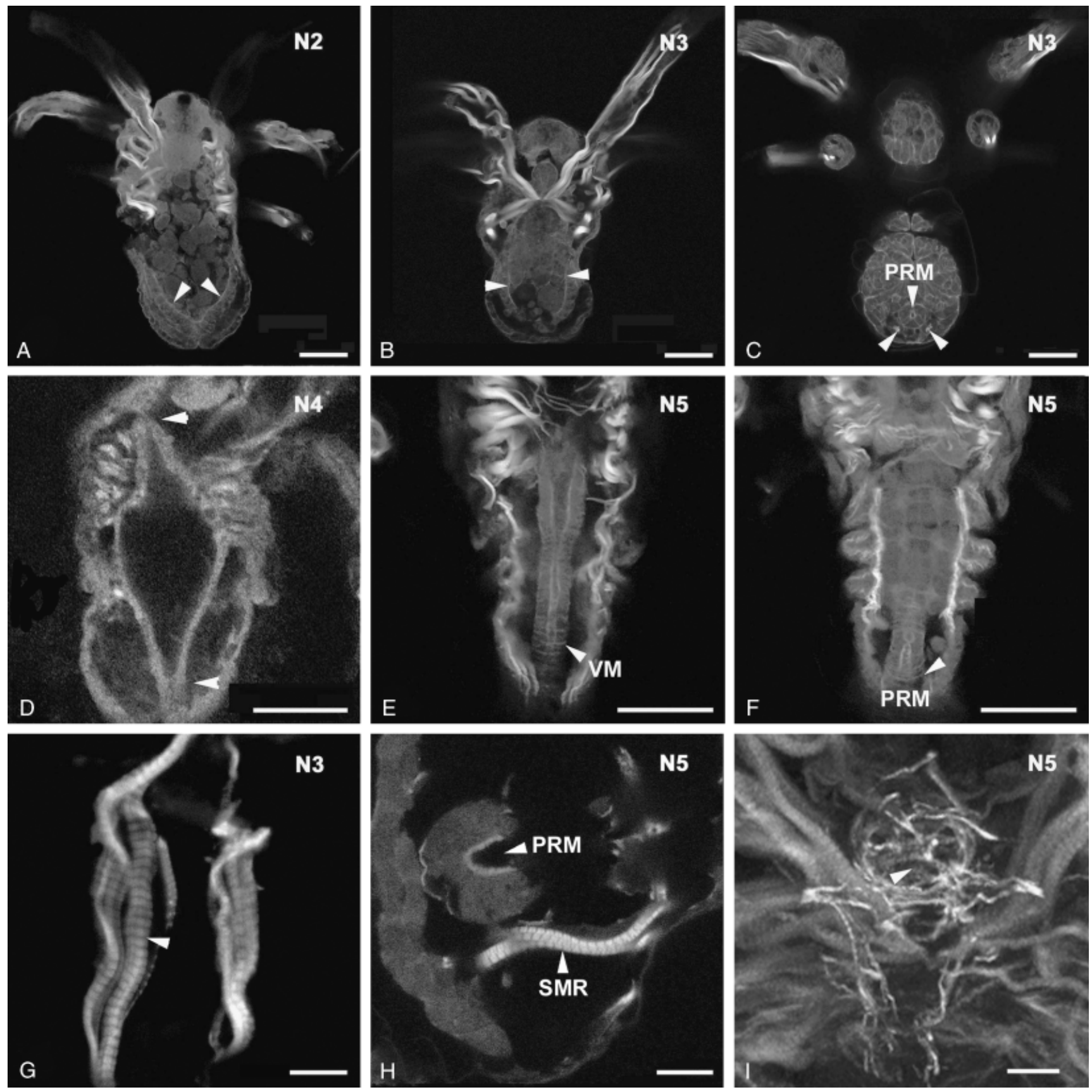

Fig. 4. Muscle development in Sicyonia nauplius larvae, single frontal optical sections of samples stained with BODIPY FL-phallacidin, oriented with anterior at the top. (A) Dorsal frontal section of a nauplius II revealing the first signs of a digestive tract (arrowheads). (B) Dorsal frontal section of a nauplius III revealing continued development of the digestive system (arrowheads). (C) Ventral frontal section of a nauplius III showing the proctodeum (PRM) with staining points to the left and right. (D) Dorsal frontal section of a nauplius IV with the midgut narrowed into a tube at the anterior and posterior-most areas (arrowheads). (E) Dorsal frontal section of a nauplius $\mathrm{V}$ with a complete digestive tube surrounded by circumferential visceral muscle (VM). (F) Mid-dorsal frontal section of a nauplius V showing the digestive tract connecting with the PRM. (G) Higher magnification view showing nauplius III limb muscle striations in ribbon-like muscles (arrowhead). (H) Higher magnification ventral frontal section of a nauplius V revealing the sarcomere (SMR) pattern of longitudinal muscle around the proctodeum (PRM). (I) The anterior region of the same nauplius $\mathrm{V}$ larvae shows striated muscle around the oral cavity (arrowhead). The sarcomere length is $1.8 \mu \mathrm{m}$. Scale bars $=50 \mu \mathrm{m}$ in A-F, $10 \mu \mathrm{m}$ in $\mathrm{G}-\mathrm{I}$. 
oral region (OR) had developed further (Fig. 3E). At $70 \mathrm{~h}$, a dorsal section revealed the developing digestive tract in the nauplius IV, which narrowed into a tube at the anterior and posterior ends (Fig. 4D, arrowheads). In the nauplius $\mathrm{V}$ at $84 \mathrm{~h}$, longitudinal muscle continued to form along the periphery of the trunk region into the furcal process (Fig. 3F, FP). Small ELM extended into the maxillae and maxillipeds. Dorsal sections showed the mature digestive tract (Figs 4, E and F), surrounded by circumferential visceral muscle (VM) and gut extension down to the PRM. Sarcomeres were clearly present in later stages (Figs 4, G-I), and only one sarcomere length was found, of $1.8 \mu \mathrm{m}$. No muscles were observed with a longer sarcomere length.

\section{Muscle development in Artemia nauplius I-metanauplius IV}

We were interested in how the Sicyonia nauplius muscle pattern compared with nonmalacostracan nauplius larvae, so Artemia nauplius I and metanauplius I-IV stages (according to Benesch 1969; Criel 1991) were also stained with BODIPY FL-phallacidin and examined by confocal microscopy. The Artemia nauplius I had extensive limb and trunk muscle development in the naupliar segments (Fig. 5A). In addition, the newly hatched nauplius had a well-developed gut, open at the stomodeum with the midgut extending halfway into the postmandibular region, whereas the hindgut (HG) was open at the proctodeum and extended anteriorly. VM surrounded the midgut in the naupliar segments.

The metanauplius I trunk was more elongated and had formed VM around the posterior hindgut invagination (Fig. 5B, VM). A dorsal section showed that epithelial cells still separated the lumens of the anterior and posterior midgut tubes (Fig. 5D). A composite of ventral sections showed oral longitudinal (OLM) and oral circumferential (OCM) muscle around the developing oral region (Fig. 5E). In the later metanauplius I, the naupliar muscles had thickened into ribbonlike sheets (Fig. 5C). A composite of dorsal sections revealed the origins of the dorsal extrinsic limb muscles (Fig. 5F), whereas a composite of ventral sections revealed the origins of the ventral extrinsic limb muscles (Fig. 5G). The gut had widened and circumferential visceral musculature (VM) was present around its entire length. At this time, the separation between anterior and posterior gut lumens was gone (Fig. $5 \mathrm{H}$, arrowhead). A composite of ventral-most sections showed the oral region with circumferential muscle (OCM) and longitudinal muscle (OLM) extension toward the posterior (Fig. 5I).

In the metanauplius II, the postmandibular region had further extended (Fig. 5J). No changes were seen in the naupliar muscles, except for elongation due to the extending limbs and trunk. The metanauplius III had maxillae and the postmaxillary region had elongated further (Fig. 5K). Thickening of longitudinal muscle was observed in all three appendages.
The metanauplius IV had developed thoracopods $(\mathrm{TH})$ and increased in length while thinning out (Fig. 5L). A sarcomere length of $1.5-2 \mu \mathrm{m}$ was consistently observed in several different muscles. No muscles were observed with longer sarcomere length.

\section{Comparison of Sicyonia and Artemia orthonauplius dorsal muscles}

Several Sicyonia nauplius I were analyzed by three-dimensional reconstruction to identify individual muscles. The Sicyonia dorsal extrinsic muscle pairs extended from bilateral positions on the dorsal carapace (Fig. 6A). The two A1 muscle pairs A1 D1 and A1 D2 originated from positions at the $\mathrm{A} 2 / \mathrm{Mn}$ boundary and inserted distally in the A1 limb. Eight A2 dorsal muscle pairs (A2 D1-8) extended in a complex pattern into the $\mathrm{A} 2$ limbs. A2 D1 originated at the same spot as A1 dorsal muscles and ran into the anterior A2 endopod. A2 D2-8 originated from bilateral positions at the posterior Mn trunk. A2 D2 ran just posterior to A2 D1, ending at the anterior joint of the appendage. A2 D3 extended posteriorly to A2 D2 within the A2 trunk and then crossed over A2 D1 ventrally to run anteriorly to A2 D1 within the A2 endopod. A2 D4 was a large muscle that inserted at the base of A2. Four muscles (A2 D5-8) were associated with the A2 exopod. Six pairs of small muscles (Mn D1-6) extended into the Mn appendages.

Artemia nauplius I were also analyzed by three-dimensional reconstruction to identify individual muscles. The muscle terminology is that used by Manton (1964) and Fryer (1983) for branchiopod and other crustacean taxa; reference is also made to the terms used by Benesch (1969) in later stage Artemia nauplii. No A1 dorsal muscles were present in the Artemia nauplius I (Fig. 6B). A series of nine A2 dorsal muscles (A2 D1-9) were found to correspond to a similar series in Branchinecta (Fryer 1983). The anterior muscles A2 D1 and A2 D2 ran from an origin on the midline of the carapace (Fig. $6 \mathrm{~B}$, position A) to insertions in the protopod. A2 D3-8 ran from an origin further posterior on the carapace midline (Fig. $6 \mathrm{~B}$, position $\mathrm{B}$ ) to various insertions in the $\mathrm{A} 2$ limbs. A2 D3 and D4 inserted at the anterior base of A2, A2 D5, and D7 inserted at the distal tip of the endopod, and A2 D6 and D8 inserted into the endopod. A2 D9 ran from a third, further posterior origin on the carapace midline (Fig. 6B, position C) and inserted into the endopod. Five sets of dorsal extrinsic muscles were found in the mandible, equivalent to muscles in Branchinecta (Fryer 1983). The anterior-most extrinsic mandibular palp muscle (EMP1) ran from its origin at position B to the distal end of the mandible. EMP2 originated from position $\mathrm{C}$ and inserted at the distal end of the mandible, near EMP1. The accessory promoter roller (AP) also ran from position $\mathrm{C}$ to insert at the distal end of the mandible, paralleling EMP2 along its entire length. Finally, two sets of 

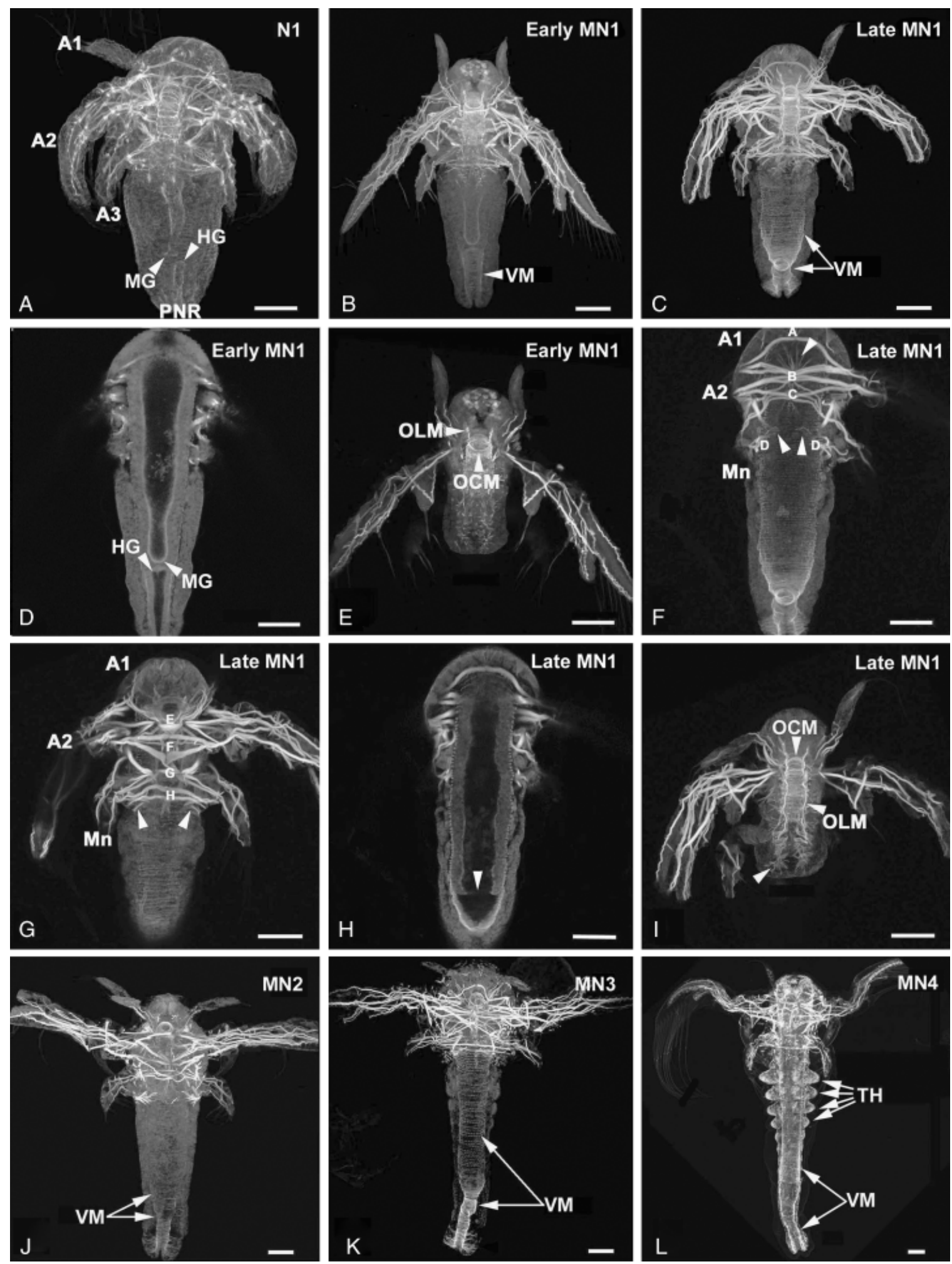


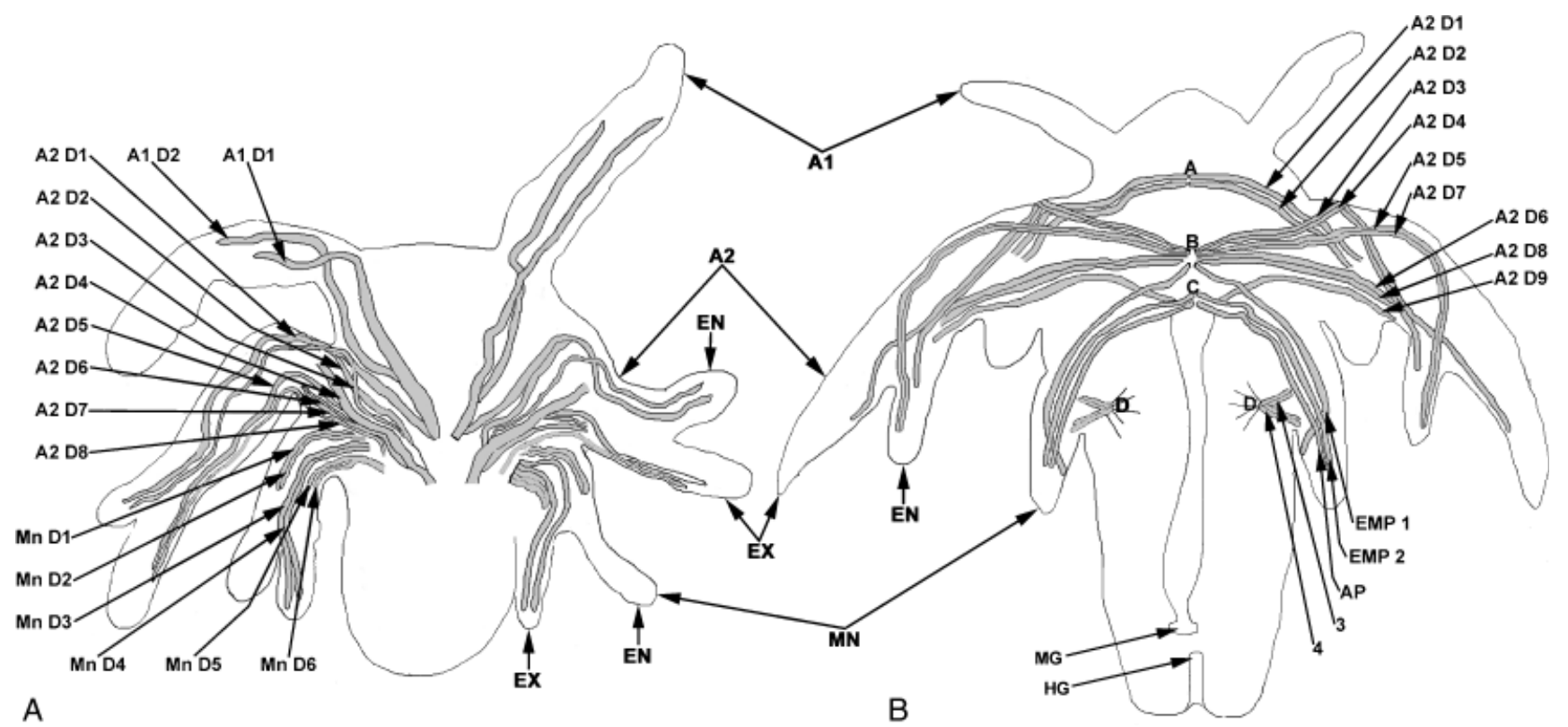

Fig. 6. Comparison of dorsal extrinsic limb muscles in Sicyonia and Artemia nauplii. (A) Sicyonia nauplius I. The dorsal A1 muscles originate from bilateral positions at the A2/Mn boundary and insert distally in the A1 limb. A2 D1 inserts into the A2 endopod. A2 D2-7 muscles originate from bilateral positions at the posterior $\mathrm{Mn}$ segment, and insert in various locations distally. A2 D8 originates from bilateral positions $\mathrm{C}$ and inserts distally on the posterior margin of the A2 exopod. Mn D1-6 muscles originate from bilateral insertion sites (D) with Mn D1 and D2 running together through the Mn trunk to the anterior base of the Mn limb. Mn D3 and D4 run together and then split extending distally into the exopod, whereas Mn D5 and D6 attach together at the posterior base of the of the Mn appendage. (B) Artemia nauplius I, using nomenclature from Fryer (1983). No dorsal muscles run to A1 in the Artemia nauplius I. A series of nine A2 dorsal muscles (D1-9) were found, originating from locations A, B, and C. Five sets of dorsal extrinsic muscles were found in the mandible. The anterior-most extrinsic mandibular palp muscle (EMP1) originates from position B and inserts at the distal end of the mandible. EMP2 and the accessory promotor roller (AP) originate from position C and insert at the distal end of the mandible, near EMP1. Finally, two sets of short muscles originate at bilateral positions D and insert in the Mn. The promotor roller muscle pair (3) runs toward the anterior of the $\mathrm{Mn}$, whereas the remotor roller muscle pair (4) runs toward the posterior. Abbreviations: AP, accessory promotor muscle of mandible; EMP, extrinsic extensor muscles of mandibular palp; HG, end of posterior midgut/hindgut; MG, end of anterior midgut; 3, mandibular promotor muscle; 4, mandibular remoter muscle; others as in previous figures.

short muscles ( 3 and 4 ) originated at bilateral positions ( $D$ in Fig. 6B) and inserted in the Mn. The two promotor roller muscles (3) ran toward the anterior of the $\mathrm{Mn}$, whereas the two remotor roller (4) muscles ran toward the posterior.

\section{Comparison of Sicyonia and Artemia orthonauplius ventral muscles}

Four pairs of ventral muscles were present in the first antennae of Sicyonia (Fig. 7A). The ventral A1 muscles (A1 V1-4

Fig. 5. Muscle development in Artemia nauplius I-metanauplius IV. Larvae were stained with BODIPY FL-phallacidin and oriented with anterior at the top. A-C and J-L are composites of optical sections through entire larvae, whereas E, F, G, I are composites of several sections, and D and $\mathrm{H}$ are single optical sections. (A) Newly hatched nauplius I, showing extensive somatic and visceral muscle (VM) in the trunk, foregut and developing midgut (MG), and hindgut (HG). (B) Early metanauplius I, showing proliferation of trunk muscle and development of posterior VM. (C) Late metanauplius I, revealing proliferation of somatic and VM. (D) Dorsal section from an early metanauplius I with anterior midgut (MG) and posterior midgut (HG) tubes still separated. (E) Ventral composite from an early metanauplius I showing longitudinal (OLM) and circular (OCM) muscle around the developing oral region. (F) Dorsal section composite from a late metanauplius I showing three dorsal muscle origins (positions A-C) extending into the A2 and Mn appendages, and a fourth, bilateral muscle origin (position D, arrowheads) extending into the Mn appendages. (G) Ventral section composite from the same late metanauplius I as F, showing muscle origin positions $\mathrm{E}$ (post-esophageal bar), F (median binding ligament), G (TMT), and H (median binding ligament). (H) Dorsal section from a late metanauplius I showing the open midgut tube (arrowhead). (I) Ventral composite from a late metanauplius I showing the oral region with circumferential muscle (OCM) and longitudinal (OLM) muscle extension toward the posterior and proliferation of muscle just below (arrowhead). (J) Metanauplius II, showing thinning and elongation of the trunk region and post-naupliar developing digestive tract surrounded by VM. (K) Metanauplius III, with more extensive somatic and VM around the elongated gut. (L) Metanauplius IV, with thoracopods (TH) and a thin, elongated body. Scale bar $=50 \mu \mathrm{m}$. 


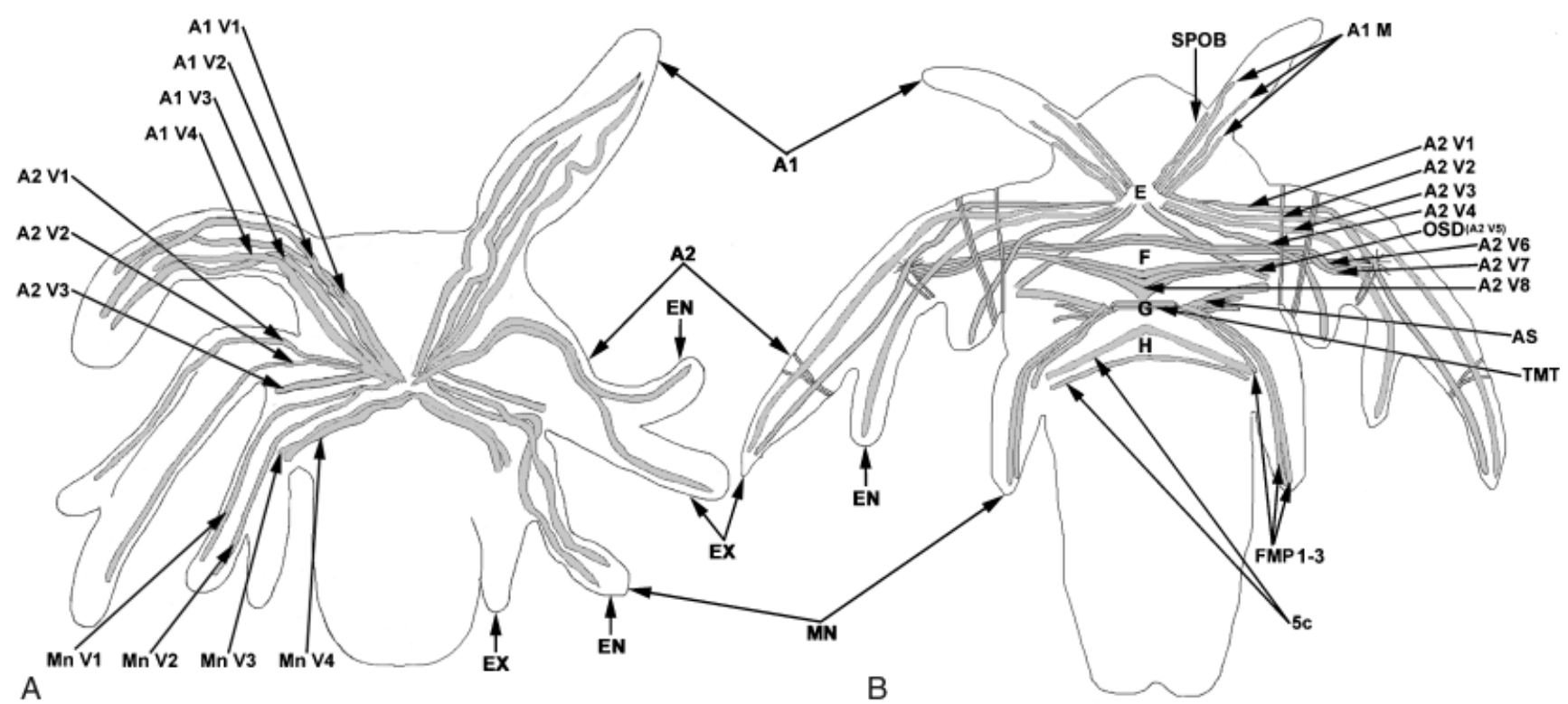

Fig. 7. Comparison of ventral extrinsic limb muscles in Sicyonia and Artemia nauplii. Homologous A1 muscles are shaded blue; A2 muscles are red; and Mn muscles are green. (A) Sicyonia nauplius I. The ventral A1 muscles, A1 V1-4, originate from a median post-esophageal location (E) and insert distally in the A1 limb. A2 V1 and V2 originate from position $\mathrm{E} / \mathrm{G}$ and insert in the distal endopod and exopod. A2 V3 (AS) runs to the posterior base of the A2 appendage. Mn V1-2 originate from position E/G, inserting in the Mn endopod. Mn V3 and Mn V4 (5c) originate even further posterior (position H), inserting at the posterior base of the Mn. (B) Artemia nauplius I, using nomenclature from Fryer (1983). The first antenna contains four ventral muscle pairs. The anterior muscle corresponds to the suspensor of the post-esophageal bar (SPOB). All four A1 muscles originate from the post-esophageal bar at position E. Eight ventral muscle pairs, designated V1-8, extend into the A2 limbs. V1-4 originate at the post-esophageal bar and insert at various locations. The esophageal suspensor dilator (OSD, A2 V5) originates at the posterior base of A2 and inserts at the esophagus. V6, V7, and V8 originate on the medial binding ligament around position F. The anterior muscles of the mandible originate on the TMT at position G. The anterior suspensor AS runs from the TMT to insert at the base of A2, near the insertion of V5. The extrinsic flexor muscles of the mandibular palp (FMP1-3) also originate from the TMT. The main posterior Mn muscle pair $(5 \mathrm{c})$ runs transversely from two origins along the medial binding ligament posterior to the TMT (position H) to insert at the posterior base of Mn. Abbreviations: A1M, first antennal muscles; AS, anterior suspensory ligament; EN, endopod; EX, exopod; FMP, extrinsic flexor muscle of mandibular palp; OSD, suspensor/dilator of esophagus; POB, post-esophageal bar; SPOB, suspensor of esophageal bar; TMT, transverse mandibular tendon; VLM, ventral longitudinal muscle; $5 \mathrm{c}$, transverse mandibular muscles; others as in previous figures.

from anterior to posterior) originated from a median postesophageal location on the ventral surface of the A2 trunk. All four A1 ventral muscles extended into the A1 limb and inserted on the exoskeleton distally. Two A2 ventral muscle pairs (A2 V1-2 from anterior to posterior) extended into the biramous A2 appendages, originating from a spot slightly posterior to the $\mathrm{A} 1$ origin. $\mathrm{A} 2 \mathrm{~V} 3$ ran to the posterior base of the A2 appendage. Four pairs of ventral muscles ran to the biramous Mn appendages (Mn V1-4), originating from a median spot slightly posterior to the A2 muscle origins.

The first antennae of Artemia contained four ventral muscle pairs, designated A1 M(An1) by Benesch (1969). The anterior muscle probably corresponds to the suspensor of the post-esophageal bar (SPOB) in Branchinecta (Fryer 1983). All four Artemia A1 muscles originated from the post-esophageal bar, equivalent to Q(An2) in Benesch (1969), in Fig. 7B, position $\mathrm{E}$. V1 and V4 inserted at the base of $\mathrm{A} 1$ whereas V2 and V3 inserted in the protopod. Eight A2 ventral muscle pairs extended into the A2 limbs. A2 V1-4 originated at the postesophageal bar and inserted at various locations. A2 V1 and A2 V2 inserted at the distal exopod, whereas A2 V3 inserted at the distal endopod. A2 V4 inserted at the ventral protopod and OSD (A2 V5), the suspensor/dilator of the esophagus, inserted at the posterior base of A2. A2 V6 and A2 V7 originated on the medial binding ligament (Fig. 7B, position F), posterior to the $\mathrm{POB}$, and ran together to the proximal exopod, whereas A2 V8 originated further posterior on the medial binding ligament and inserted at the proximal exopod, near the insertion of A2 V6/7.

The anterior muscles of the mandible originated on the transverse mandibular tendon (TMT), equivalent to $\mathrm{Q}(\mathrm{Md})$ in Benesch (1969), in Fig. 7B, position G. The anterior suspensor (AS) originated at the TMT and inserted at the base of A2, near V5. The extrinsic flexor muscles of the mandibular palp, FMP1-3, also originated from the TMT; FMP1 and 2 inserted at the distal tip of Mn whereas FMP3 inserted near the 
Table 1. Muscles in crustacean nauplius larvae

\begin{tabular}{|c|c|c|c|c|}
\hline \multirow{2}{*}{$\begin{array}{l}\text { Taxon } \\
\text { Species } \\
\text { (Reference) }\end{array}$} & \multicolumn{2}{|c|}{ Branchiopoda: Anostraca } & \multirow{2}{*}{$\begin{array}{l}\text { Maxillopoda: Cirrepedia } \\
\text { Balanus } \\
\text { (Walley 1969) }\end{array}$} & \multirow{2}{*}{$\begin{array}{l}\text { Malacostraca: Decapoda: Dendrobranchiata } \\
\text { Sicyonia } \\
\text { (Present study) }\end{array}$} \\
\hline & $\begin{array}{l}\text { Branchinecta } \\
\text { (Fryer 1983) }\end{array}$ & $\begin{array}{l}\text { Artemia } \\
\text { (Present study) }\end{array}$ & & \\
\hline Dorsal A1 extrinsic & 0 & 0 & 4 & 2 \\
\hline Ventral A1 extrinsic & 3 & 4 & 1 & 4 \\
\hline Dorsal A2 extrinsic & 9 & 9 & 10 & 8 \\
\hline Ventral A2 extrinsic & 8 & 8 & 6 & 3 \\
\hline Dorsal Mn extrinsic & 4 & 4 & 5 & 4 \\
\hline Ventral Mn extrinsic & 5 & 5 & 3 & 4 \\
\hline A2 intrinsic & 6 & 6 & $?$ & 0 \\
\hline
\end{tabular}

posterior base of $\mathrm{Mn}$. If the uniramous mandibular palps are considered to be endopodites, then Sicyonia Mn V1 and V2 may correspond to Artemia FMP1 and 2. The main posterior Mn muscle $5 \mathrm{c}$ ran from two origins along the medial binding ligament posterior to the TMT (Fig. 7B, position $\mathrm{H}$ ) to insert at the posterior base of the mandible.

\section{DISCUSSION}

\section{Naupliar muscle development in Malacostraca: Dendrobranchiata}

We used phallotoxin staining to stain the muscles of Sicyonia embryos and nauplius larvae. Although other cell types may also contain F-actin, only muscle cells have the characteristic striated appearance from the sarcomeres. More specific markers such as muscle-specific transcription factors would prove useful for identifying prospective muscle cells earlier in development. Muscle development was followed in Sicyonia from late embryogenesis through nauplius V. Whereas F-actincontaining structures were first detected in Sicyonia embryos at $12 \mathrm{~h}(50 \%$ development to hatching), fibers containing striations were first observed at $19 \mathrm{~h}(80 \%$ development to hatching). We interpret the early F-actin staining to be in nerve cells associated with the spines on the limbs and the F-actin staining with striations to be muscle.

The nauplius hatched with the following pairs of extrinsic limb muscles (summarized in Table 1): two dorsal A1, four ventral A1, eight dorsal A2, three ventral A2, four dorsal Mn, and four ventral Mn. These extrinsic limb muscles grew and thickened during later naupliar stages, consistent with the gradual, anamorphic development in dendrobranchiates. Longitudinal trunk muscles developed during nauplius IV and V. No attempt was made to give the naupliar muscles functional names, as they have not been traced through the remaining larval stages to the postlarva. Preliminary observations suggest that the adult muscles can be identified by the mysis stages, so that it should be possible to trace the naupliar muscles back and assign functional identities, as determined for the adult (Young 1959).

No intrinsic limb muscles or VMs were found in the Sicyonia nauplius I, and all muscles had the same sarcomere length. The nauplius stages in dendrobranchiates are nonfeeding, with nutrition coming from EYC. In development to the $P$. kerathurus ( $=P$. trisulcatus) nauplius, the endoderm forms an endothelial rudiment that is open on the dorsal side, with more anterior EYC proposed to give rise to the digestive gland (Zilch 1979). Further development of the gut during the naupliar stages was observed in Sicyonia: the gut formed as a single tube, initially constricted in the anterior and posterior midgut regions, during nauplius IV. VMs were observed around the gut in the nauplius $\mathrm{V}$ in preparation for feeding at the subsequent protozoeal stages. In Penaeus (Farfantepenaeus) aztecus, the midgut was found to be fully differentiated at the ultrastructural level in protozoa I (Talbot et al. 1972).

\section{Naupliar muscle development in Branchiopoda: Anostraca}

The muscle pattern of Artemia was very similar to that described from histological sections from Branchinecta (Fryer 1983). Similar numbers of muscles were found in the two Branchiopoda species (Table 1), except that three ventral A2 extrinsic muscles were present in Branchinecta whereas four were found in Artemia. Homologous muscles were identified, based on the following criteria for assigning muscle homologies (Laubichler 2000, after Remane 1952): (1) similar position including segment location, anterior-posterior, dorsal-ventral, and proximal-distal, (2) similar structural details, including origin and insertion sites and intermediate attachments, and (3) similar transitional forms throughout subsequent larval instars. VMs were found surrounding the gut in the Artemia nauplius I., although the midgut tubes had not yet joined. Full function of the digestive tract would not be possible until fusion of the two midgut invaginations at the late metanauplius stage, and feeding occurs in these later nauplius stages. 


\section{Comparison of naupliar development in the Crustacea}

The present study suggests that different patterns of extrinsic limb muscles are present in crustacean nauplii. As summarized in Table 1, the numbers of muscles are different in the Branchiopoda, Cirrepedia, and Dendrobranchiata. It seems likely that the loss and/or gain of naupliar limb muscles, for example in the A1, correlates with the use of the limbs for swimming or feeding. Comparison of the muscle pattern of dendrobranchiate and branchiopod nauplii (Figs. 6 and 7) with a cirrepede nauplius (Walley 1969, Figs. 4 and 5) shows different origins for the extrinsic limb muscles as well as variation in the overall pattern. Whereas homologous muscles can clearly be identified in the two anostracan branchiopods $\mathrm{Ar}$ temia and Branchinecta, the identification of homologous muscles in dendrobranchiates or cirripedes cannot be made at present.

The endoderm also develops differently in branchiopod and dendrobranchiate nauplii. In Sicyonia, the gut tube is not present in the first naupliar stage and forms during stages IV and $\mathrm{V}$ as a single tube that narrows from either end. The VMs form later in consequence and EYC are still present in naupliar stages. In Artemia, the anterior and posterior midgut tubes are already present in the first naupliar stage, along with associated VM. The septum that blocks the gut opens during the second naupliar stage, the metanauplius I. Therefore, this study has identified differences in both somatic muscle pattern and endoderm development between malacostracan and nonmalacostracan nauplii. These differences are consistent with the secondary evolution hypothesis of the dendrobranchiate-FN.

The present study cannot resolve how many times the FN evolved in the Malacostraca, or the overall relationships of the major crustacean groups. The range of crustacean FN larvae for which muscle anatomy can be compared is still small. An obvious follow-up study would be to examine the naupliar muscle pattern in the Euphausiacea. If the FN evolved once among the ancestors of the Dendrobranchiata and Euphausiacea, then the naupliar muscle patterns would be predicted to be similar. If the FN evolved independently in the Dendrobranchiata and Euphausiacea, then the naupliar muscle patterns would be predicted to be less similar. It would also be of interest to see how the endoderm develops in the two groups. A recent study has proposed homologies of cleavage and gastrulation in Dendrobranchiata and Euphausiacea (Alwes and Scholtz 2004). This would seem to support a sister relationship between the Euphausiacea and Decapoda. However, other studies support a sister relationship between the Euphausiacea and Peracarida (Jarman et al. 2000; Richter and Scholtz 2001). Further studies are needed to better understand the evolution of development in the Malacostraca and in the Crustacea in general.

\section{Acknowledgments}

We are grateful to Dr. Gary Martin (Occidental College) for use of his lab facilities, and to the crew of the R/V Vantuna for shrimp collection. Thanks are due to Osvaldo Jhonatan Sepulveda Villet, Central Michigan University, for technical assistance. Thanks are especially due to Drs. Terri Williams (Yale University), Jennifer Schisa and Bradley Swanson (Central Michigan University), and two anonymous reviewers for their suggestions on improving various drafts of the manuscript. This work was supported by the Department of Biology, College of Graduate Studies, and College of Science and Technology at Central Michigan University; State of Michigan/ Central Michigan University Research Excellence Funds; and CMU President's Research Initiative Funds.

\section{REFERENCES}

Alwes, F., and Scholtz, G. 2004. Cleavage and gastrulation of the euphausiacean Meganyctiphanes norvegica (Crustacea, Malacostraca). Zoomorphology 123: 125-137.

Anderson, D. T. 1969. On the embryology of the cirripede crustaceans Tetraclita rosea (Krauss), Tetraclita purpurascens (Wood), Chthamalus antennatus (Darwin) and Chamaesipho columna (Spengler) and some considerations of crustacean phylogenetic relationships. Phil. Trans. R. Soc. B 256: 183-235.

Anderson, D. T. 1973. Embryology and Phylogeny of Annelids and Arthropods. Pergaman Press, Oxford.

Anderson, D. T. 1994. Barnacles: Structure, Function, Development and Evolution. Chapman \& Hall, London.

Benesch, R. 1969. Zur Ontogenie und Morphologie von Artemia salina. Zool. Jb. Anat. Ont. 86: 307-458.

Bowler, P. J. 1994. Are the Arthropoda a natural group? An episode in the history of evolutionary biology. J. Hist. Biol. 27: 177-213.

Cavanaugh, G. M. (Ed.). 1956. Formulae and Methods of the Marine Biological Laboratory Chemical Room. 6th Ed. Marine Biological Laboratory, Woods Hole, MA.

Criel, G. R. J. 1991. Ontogeny of Artemia. In R. A. Browne, P. Sorgeloss, and C. N. Trotman (eds.). Artemia Biology. CRC Press, Boca Raton, FL, pp. 155-185.

Dahms, H.-U. 2000. Phylogenetic implications of the crustacean nauplius. Hydrobiologia 417: 91-99.

Dall, W., Hill, B. J., Rothlisberg, P. C., and Sharples, D. J. 1990. The biology of the Penaeidae. In J. H. S. Blaxter and A. J. Southward (eds.) Advances in Marine Biology. Vol. 27. Academic Press, London.

Daniel, R. J. 1930. The abdominal muscular systems of the zoëa and mysis stages of the shrimp (Crangon vulgaris Fabr.) and their bearing on phylogeny. Proc. Trans. Liverpool Biol. Soc. 44: 95-109.

Fryer, G. 1983. Functional ontogenetic changes in Branchinecta ferox (Milne-Edwards) (Crustacea: Anostraca). Phil. Trans. R. Soc. London B 303: 229-343.

Hertzler, P. L. 2002. Development of the mesendoderm in the dendrobranchiate shrimp Sicyonia ingentis. Arthropod Struct. Dev. 31: 33-49.

Hertzler, P. L., and Clark, W. H. Jr. 1992. Cleavage and gastrulation in the shrimp Sicyonia ingentis: invagination is accompanied by oriented cell division. Development 116: 127-140.

Hessler, R. R. 1964. The Cephalocarida: comparative skeletomusculature. Mem. Connecticut Acad. Arts Sci. 16: 1-97.

Jarman, S. N., Nicol, S., Elliott, N. G., and McMinn, A. 2000. 28S rDNA evolution in the Eumalacostraca and the phylogenetic position of krill. Mol. Phyl. Evol. 17: 26-36.

Laubichler, M. D. 2000. Homology in development and the development of the homology concept. Am. Zool. 40: 777-788.

Manton, S. M. 1964. Mandibular mechanisms and the evolution of arthropods. Phil. Trans. R. Soc. London B 247: 1-183.

Manton, S. M. 1977. The Arthropoda: Habits, Functional Morphology, and Evolution. Clarendon Press, Oxford.

Müller, F. 1864. Für Darwin. Engelmann, Leipzig. 
Pillai, M. C., Griffin, F. J., and Clark, W. H. Jr. 1988. Induced spawning of the decapod crustacean Sicyonia ingentis. Biol. Bull. 174: 181-185.

Remane, A. 1952. Die Grundlagen des natürlichen Systems, der vergleichenden Anatomie und der Phylogenetik. Akademische Verlagsgesellschaft, Leipzig.

Richter, S., and Scholtz, G. 2001. Phylogenetic analysis of the Malacostraca (Crustacea). J. Zool. Syst. Evol. Res. 39: 113-136.

Scholtz, G. 2000. Evolution of the nauplius stage in malacostracan crustaceans. J. Zool. Syst. Evol. Res. 38: 175-187.

Strathmann, R. R., and Eernisse, D. J. 1994. What molecular phylogenies tell us about the evolution of larval forms. Am. Zool. 34: 502-512.

Talbot, P., Clark, W. H. Jr., and Lawrence, A. L. 1972. Fine structure of the midgut epithelium in the developing brown shrimp, Penaeus aztecus. $J$. Morphol. 138: 467-486.

Walley, J. L. 1969. Studies on the larval structure and metamorphosis of Balanus balanoides (L). Phil. Trans. R. Soc. London B 236: $237-280$
Walossek, D. 1999. On the Cambrian diversity of Crustacea. In F. R. Schram and J. C. Vaupel Klein (eds.). Crustaceans and the Biodiversity Crisis-Proceedings of the Fourth International Crustacean Congress. Brill, Leiden, Amsterdam, pp. 3-27.

Williams, T. A. 1994. The nauplius larva of crustaceans: functional diversity and the phylotypic stage. Amer. Zool. 34: 562-569.

Williams, T. A., and Müller, G. B. 1996. Limb development in a primitive crustacean, Triops longicaudatus: subdivision of the early limb bud gives rise to multibranched limbs. Dev. Genes Evol. 206: 161-168.

Young, J. H. 1959. Morphology of the white shrimp Penaeus setiferus. United States Department of the Interior, Fish and Wildlife Service. Fishery Bull. 59: 1-168.

Zilch, R. 1978. Embryologische Untersuchungen an der holoblastischen Ontongenese von Penaeus trisulcatus Leach (Crustacea, Decapoda). Zoomorphologie 90: 67-100.

Zilch, R. 1979. Cell lineage in arthropods? Fortschr. Zool. Syst. Evol. Forsch. 1: 19-41. 